\title{
ON THE CENTRALIZER OF A SUBGROUP OF A LIE GROUP
}

\author{
DONG HOON LEE ${ }^{1}$ \\ Abstract. In this paper, a theorem on the centralizer of a \\ closed subgroup $H$ of a Lie group $G$ such that $G / H$ admits a finite \\ invariant measure is proved.
}

Let $G$ be a connected Lie group and $H$ a closed subgroup of $G$ such that the homogeneous space $G / H$ of left cosets admits a positive invariant measure $\mu$. The main purpose of this paper is to prove the following theorem.

Theorem. If $\mu(G / H)$ is finite, then the closure of the commutator subgroup of the centralizer of $H$ in $G$ is compact.

Motivated by the density theorem of Borel [1], Wang [6] has obtained the above-mentioned result, when $H$ is discrete. In $\$ 1$, we collect some of the basic facts on measure theory. $\$ 2$ carries the complete proof of the announced theorem.

Finally, I would like to express my gratitude to Professor T. S. Wu for a useful conversation during the preparation of this paper.

1. Invariant measures on homogeneous spaces. In this section, we summarize some measure theoretic results which will be needed later. For more details, see [4] and [7].

For a locally compact space $X$, let $C_{c}(X)$ denote the space of all real valued continuous functions on $X$ with compact support. Then a positive measure on $X$ is a real valued linear functional $\mu: C_{c}(X) \rightarrow R$, such that $f \in C_{c}(X)$ is nonnegative; then $\mu(f) \geqq 0$.

Suppose now that $G$ is a locally compact topological group and $H$ is a closed subgroup of $G$. Given any $\varphi \in C_{c}(G / H)$ and $a \in G$, let ${ }_{a} \varphi$ be defined by ${ }_{a} \varphi(x H)=\varphi(a x H), x H \in G / H$. Then a positive measure $\mu$ on $G / H$ is called relatively invariant (relative to the multiplier $\chi$ ) if

(1) $\mu\left({ }_{a} \varphi\right)=\chi(a) \mu(\varphi)$ for all $a \in G$ and $\varphi \in C_{c}(G / H)$, and

(2) $\chi$ is a homomorphism of $G$ in to the multiplicative group $R^{+}$of positive real numbers.

Received by the editors February 9, 1970.

AMS 1969 subject classifications. Primary 2210, 2250; Secondary 2290.

Key words and phrases. Homogeneous space, invariant measure, Borel's density theorem, compact support, multiplier, Haar measure, lattice, compactly generated $[F C]^{-}$-group, compact element, periodic subgroup.

1 This research was supported in part by NSF Grant GP 12261. 
If the multiplier $\chi$ is trivial, then we simply say that $\mu$ is an invariant measure. A relatively invariant measure is uniquely determined by its multiplier up to a constant factor. When $H$ is an invariant closed subgroup of $G$, then an invariant measure is easily seen to be a Haar measure.

If $H$ is a discrete subgroup of a Lie group $G, H$ is called a lattice when a measure $\mu$, induced on the homogeneous space $G / H$ by a left invariant Haar measure on $G$, satisfies $\mu(G / H)<\infty$. If $G$ contains a lattice $H$, then $G$ is a unimodular group. If this is the case, then $\mu$ is an invariant measure.

We close this section with the following lemma due to G. D. Mostow [4, p. 22] which will be repeatedly used later.

Lемма. Let $G$ be a locally compact group and $H \subseteq F$ be closed subgroups of $G$. If $G / H$ has a finite invariant measure $\mu$, then $G / F$ and $F / H$ both admit finite invariant measures of which $\mu$ is a product.

2. Proof of the theorem. Throughout this section, $L_{0}$ for any Lie group $L$ stands for the identity component of $L$. We begin with the following lemma ${ }^{2}$ whose proof may be found in the appendix of [5]:

LEMmA A. Let $G$ be a connected Lie group with its radical $R, \pi: G$ $\rightarrow G / R$ the projection and $K$ a closed subgroup of $G$. If $K_{0}$ is solvable, then the identity component of the closure $\operatorname{cl}(\pi(K))$ of $\pi(K)$ is solvable.

LEMMA B. Every closed subgroup of a connected solvable Lie group is compactly generated.

Proof. Let $K$ be a closed subgroup of a connected solvable Lie group $L, \tilde{L}$ the simply connected covering group of $L$, and let $\tilde{K}$ be the complete inverse image of $K$ under the covering projection $\sigma$.

Clearly $\sigma\left(\tilde{K}_{0}\right)=K_{0}$, and hence $\sigma$ induces an epimorphism $\tilde{K} / \tilde{K}_{0}$ $\rightarrow K / K_{0}$ of discrete groups. Since a group is compactly generated if and only if it is finitely generated modulo its identity component, it suffices to show $\tilde{K} / \tilde{K}_{0}$ is finitely generated. Hence we may assume that $L$ is simply connected. Noting that $L / K_{0}$ is simply connected, we can identify $K / K_{0}$ with the fundamental group of $L / K$. Since $K / K_{0}$ is solvable, it is finitely generated by the well-known theorem of Mostow [3]. Hence $K$ is compactly generated.

We now improve Lemma $B$ as follows:

2 The Lemma A was stated in [5] under the further assumption that $R$ is simply connected. However, it can be easily proved, using induction on the length of solvability of $R$, that this assumption can be removed. 
LEMMA C. Let $L$ be a connected Lie group whose semisimple part is compact and let $K$ be a closed subgroup such that $K_{0}$ is solvable. Then $K$ is compactly generated.

Proof. Let $R$ denote the radical of $L$. From Lemma A, it follows that $(\operatorname{cl}(K R))_{0}$ is solvable. Since $L$ is compact modulo $R, \operatorname{cl}(K R) /(\operatorname{cl}(K R))_{0}$ is finite. On the other hand, the solvability of $(\mathrm{cl}(K R))_{0}$ implies that $(\operatorname{cl}(K R))_{0} \cap K$ is compactly generated $\left(\right.$ Lemma B). Since $K / K \cap(\operatorname{cl}(K R))_{0} \cong K(\operatorname{cl}(K R))_{0} /(\operatorname{cl}(K R))_{0}$ $=\operatorname{cl}(K R) /(\operatorname{cl}(K R))_{0}$ is finite, $K$ is compactly generated.

With this preparation, we are ready to present the proof of the announced theorem. Throughout the proof, $Z$ denotes the centralizer of $H$ in $G$ and $Z^{\prime}$ the topological commutator subgroup (that is, the closure of the commutator subgroup) of $Z$.

Let $C$ be the maximal connected normal compact subgroup of a semisimple part of $G$ and let $R$ be the radical of $G$. We first show that $Z^{\prime} \subseteq R C$. In fact, let $\mathrm{cl}(H R C)$ denote the closure of the subgroup $H R C$ in $G$. Then $G / \operatorname{cl}(H R C)$ admits a finite invariant measure by the lemma of $\S 1$. Since $G / R C$ is a connected semisimple Lie group, all of whose simple factors are noncompact, we see that the centralizer of $\operatorname{cl}(H R C) / R C$ is exactly the center of $G / R C$ by the density theorem of Borel [1]. In particular, $Z$ is abelian modulo $R C$. Hence $Z^{\prime} \subseteq R C$ follows.

Next we show that $Z^{\prime}$ is an $[F C]^{-}$-group. (A topological group is called an $[F C]^{-}$-group, if the closure of each conjugacy class is compact.) To see this, consider the closure $\operatorname{cl}(Z H)$ of $Z H$. Again by the lemma of $\$ 1, \operatorname{cl}(Z H) / H$ admits a finite invariant measure, which is a left Haar measure because $H$ is an invariant subgroup of $\operatorname{cl}(Z H)$. Thus $\mathrm{cl}(Z H) / H$ is compact. Let $x \in Z$ and define $\Gamma(x)=\left\{g x g^{-1} \mid g\right.$ $\in \mathrm{cl}(Z H)\}$. Since the centralizer of $x$ contains $H$, modulo which $\operatorname{cl}(Z H)$ is compact, $\Gamma(x)$ is easily seen to be compact. Thus $Z$ (and hence $\left.Z^{\prime}\right)$ is an $[F C]^{-}$-subgroup of $G$.

By a theorem of Grosser and Moskowitz [2, Theorem (3.16)], the set of all compact elements of $Z$ forms a closed characteristic subgroup, modulo which $Z$ is abelian. Hence $Z^{\prime}$ is also a closed periodic subgroup of $Z$. (A topological group is called periodic, if every element is contained in a compact subgroup.)

As a compactly generated periodic $[F C]^{-}$-group is compact $[2$, Proposition (3.17) ], it suffices to show that $Z^{\prime}$ is compactly generated.

The compactness of the identity component $Z_{0}^{\prime}$ of $Z^{\prime}$ follows easily from the fact that $Z^{\prime}$ is periodic. Let $S$ be the semisimple part of the 
compact group $Z_{0}^{\prime}$, and let $\operatorname{Aut}(S)$ (resp. Int $(S)$ ) denote the group of automorphisms (resp. the subgroup consisting of inner automorphisms) of $S$. Then $\operatorname{Aut}(S) / \operatorname{Int}(A)$ is finite.

Since $S$ is characteristic in $Z_{0}^{\prime}$, it is an invariant subgroup of $Z^{\prime}$. Hence we can define a homomorphism $\pi: Z^{\prime} \rightarrow \operatorname{Aut}(S)$ by $\pi(x)(y)$ $=x y x^{-1}, x \in Z^{\prime}$ and $y \in S$. Then the complete inverse image of $\operatorname{Int}(S)$ under $\pi$ is of finite index in $Z^{\prime}$. Thus we may assume, without losing generality, that $\pi\left(Z^{\prime}\right)=\operatorname{Int}(S)$.

Let $A$ be the centralizer of $S$ in $Z^{\prime}$. It is then easy to see that $Z^{\prime}$ $=A S$ and that $A_{0}$ is the maximal central torus of $Z_{0}^{\prime}$. Recalling that $Z^{\prime} \subseteq R C$, it follows from Lemma $\mathrm{C}$ that $A$ is compactly generated. Thus $Z^{\prime}=A S$ is also compactly generated, which finishes our proof.

As a consequence of the theorem, we have

CoROLlARY. If $G$ is a connected simply connected Lie group such that the maximal connected compact invariant subgroup of a semisimple part of $G$ is trivial, then the centralizer $Z$ of $H$ is abelian.

Proof. We have seen, in the proof of the theorem, that $Z^{\prime} \subseteq R C$ (using the notation there). Since $G$ is simply connected, the radical $R$ has no compact subgroups. Thus the triviality of $C$ implies that $Z^{\prime}$ $=\{1\}$, which means that $Z$ is abelian.

\section{REFERENCES}

1. A. Borel, Density properties for certain subgroups of semi-simple groups without compact components, Ann. of Math. (2) 72 (1960), 179-188. MR 23 \#A964.

2. S. Grosser and M. Moskowitz, Compactness conditions in topological groups. I, J. Reine Angew. Math. (to appear).

3. G. D. Mostow, On the fundamental group of homogeneous space, Ann. of Math. (2) 66 (1957), 249-255. MR 19, 561.

4. - Homogeneous spaces with finite invariant measure, Ann. of Math. (2) 75 (1962), 17-37. MR 26 \#2546.

5. H. C. Wang, On the deformations of lattice in a Lie group, Amer. J. Math. 85 (1963), 189-212. MR 27 \#2582.

6. S. P. Wang, On the centralizer of a lattice, Proc. Amer. Math. Soc. 21 (1969), 21-23. MR 38 \#5989.

7. A. Weil, L'intégration dans les groupes topologiques et ses applications, Actualités Sci. Indust. no. 869, Hermann, Paris, 1940. MR 3, 198.

Case Western Reserve University, Cleveland, Ohio 44106 